\title{
Nonlinear observers design for multivariable ship motion control
}

\author{
Mirosław Tomera, Ph. D., \\ Gdynia Maritime University
}

\begin{abstract}
This paper presents the designs of two observers, which are: the extended Kalman filter and the nonlinear passive observer. Based on the measured values of ship position and heading, the observers estimate the surge, sway and yaw velocities of the ship motion. The observers make use of the simplified nonlinear mathematical model of ship motion in which the neglected ship dynamics and disturbances are modelled using bias. The designed observers firstly have been simulated on a computer model where their parameters were calibrated, and then were implemented on the physical model of the training ship "Blue Lady" in the ship handling centre in Ilawa-Kamionka. The comparative analysis was done with respect to the estimated variables describing the ship motion in three directions: surge, sway and yaw.
\end{abstract}

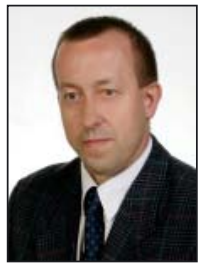

Keywords: extended Kalman filter; nonlinear observers; ship control; dynamic positioning.

\section{INTRODUCTION}

Dynamic positioning is the control system which automatically keeps the ship position and heading using propellers and thrusters installed on the ship. Correctness of operation of the ship positioning system depends on the accuracy of the adopted mathematical model. Controlling ship motion is a difficult task, due to the fact that ship dynamics are nonlinear, time varying and uncertain. Moreover, environmental disturbances such as the wind, waves, and sea currents make the control task even more difficult. During a few recent decades, various advanced methods of ship control have been developed, especially in the field of robotics, which made it possible to reach higher quality of control. The controllers developed in this field require measuring all state variables, which is impractical and in some cases even impossible.

In case of ship motion control in three degrees of freedom, the two translational velocities (longitudinal and lateral) and the angular speed are difficult for measuring, compared to the remaining states. The control systems used in dynamic positioning systems require the information on these velocities, complemented by the data on the ship position and heading. Usually the velocities are calculated from the position and heading of the ship, the measurement of which is affected by certain disturbances. That is why the filtration and state estimation play an important role in designing the dynamic positioning systems [1].

Velocity estimates are calculated from measured the ship position and heading data by the state observer. Unfortunately, these measurements are corrupted with the coloured noise generated by the wind, waves and oceanic currents, as well as the noise generated by the sensor itself. The environmental disturbances acting on the ship generate two separate movements. The sea waves of the first order generate highfrequency movements, while the slowly changing forces generate low-frequency movements. Only slowly changing disturbances are to be compensated by the propellers installed on the ship, while the oscillatory movements generated by the waves (wave disturbances of the first order) should not enter the control system loop. This strategy is executed using wave filtration techniques which divide the measured position and heading signals into the low frequency part (LF) and the high frequency part generated by the waves (WF) [2].

The dynamic positioning systems have been developed since the early sixties of the last century. The first dynamic control systems were designed using conventional PID controllers working in cascade with low-pass filters or cut-off filters to separate the motion components connected with the sea waves. However, those systems introduce phase delays which worsen the quality of the control [1].

From the middle of 1970 s more advanced control techniques started to be used, which were based on optimal control and the Kalman filter theory. The first solution of this type was presented by Balchen, Jenssen and Saelid [3]. It was then modified and extended by Balchen himself and other researchers $[4,5,6,7,8,9]$. The new solutions made use of the linear theory, according to which the kinematic characteristics of the ship were to be linearized in the form of sets of predefined ship heading angles, with an usual resolution of 10 degrees. After the linearization of the nonlinear model, the observer based on such a model is only locally correct. This is the disadvantage of the Kalman filter. 


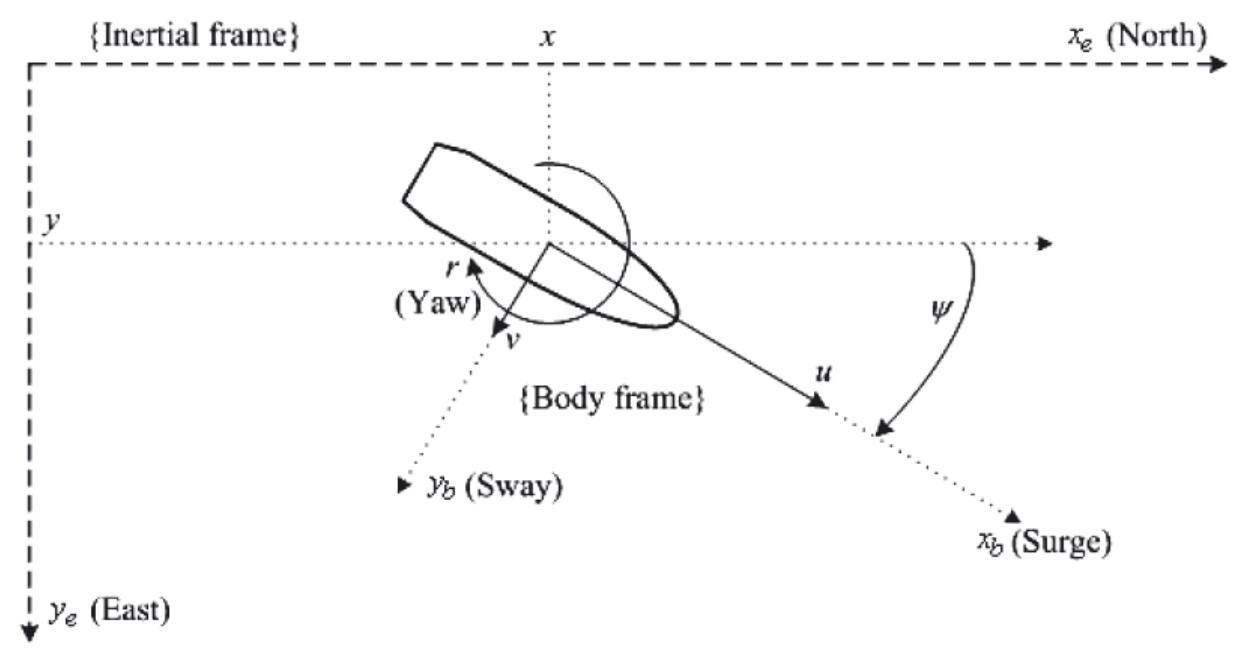

Fig. 1. Definition of the adopted coordinate systems: inertial frame (earth-fixed frame) $x_{e} y_{e} z_{e}$ and body frame (body fixed frame) $x_{b} y_{b} z_{b}$ connected with the moving ship

To avoid the linearization of the kinematic equations of ship motion, attempts have been made to apply nonlinear control to the dynamic positioning systems. Grovlen and Fossen proposed the use of vector backstepping as a possible solution to the problem of control in the dynamic positioning system. This solution based on the filtration of the measured ship position and heading signals, which were assumed to contain only the white noise as the disturbance. However, in practice these signals are also corrupted with the coloured noise connected with sea waves acting on the ship hull. Therefore to avoid excessive activity (wear and tear) of the propulsion system, the calculated velocity estimates were to be filtered, before introducing to the fedback loop, using so-called wave filtration techniques which extracted the wave frequency (WF) from the measured movements for the controller to obtain only low frequencies. The improved version of this design was presented by these same authors [10]. Those works are considered the first cases in which a fully nonlinear system of dynamic positioning was used - although this system missed wave filtration, and bias modelling and estimation to separate the non-modelled slowly changing disturbances.

Wave filtration, bias estimation, reconstruction of LF movement components, and calculating noise-free estimates of unmeasured ship velocities were taken into account by Fossen and Strand who used for this purpose a nonlinear passive observer [11]. The nonlinear observer proposed by them showed new areas for controller designing, done based on the existing structure of the physical system and making use of the principle of separation developed by [12] or the backstepping method described by [13].

Activities are being in progress upon further development of the nonlinear systems in their application to dynamic positioning systems. Kim and Inman have designed a robust nonlinear observer which makes use of the sliding mode concept. The basic advantage of this observer is its robustness connected with the neglected nonlinearities, disturbances and uncertainties. The principle of operation of this observer bases on the work by Fossen and Strand, and it delivers the estimates of the linear ship velocities and the bias describing the slowly changing environmental disturbances [14]. Snijders, van der Woude, and Westhuis have designed a suboptimal observer making use of the Riccati equation and based on the contraction theory. The authors presented theoretical principles of the optimal nonlinear observer [15]. Hajivand and Mousavizadegan have designed a nonlinear observer for wave filtering and velocity estimation. Their observer makes use of the memory effect and takes into account the dependence of the added mass and damping coefficient changes in the ship dynamics model on the oscillatory forces acting on the ship hull [16].

\section{MATHEMATICAL MODELLING OF THE SHIP}

The present work mainly aims at comparing the quality of operation of the Extended Kalman Filter (EKF) [17] with the nonlinear observer [11]. The both designs make use of the mathematical model of ship dynamics in which the ship motion is the superposition of the LF and HF movements.

\section{a) Kinematic equation of motion}

Two coordinate systems are introduced to describe the motion of a sea-going ship. The first coordinate system is connected with the moving ship and bears the name of the body-fixed frame. The centre of this system is in the centre of ship's gravity. The motion of the body-fixed frame is described with respect to the stationary coordinate system called the earth-fixed frame (Fig. 1). The ship position (x, y) and the ship heading $\psi$, described with respect to the earth-fixed frame, can be expressed in the vector form as $\boldsymbol{\eta}=[\mathrm{x}, \mathrm{y}, \psi]^{\mathrm{T}}$. In the same form the velocities in the body-fixed frame can be given as $\mathbf{v}=[\mathrm{u}, \mathrm{v}, \mathrm{r}]^{\mathrm{T}}$.

The relation between the vessel-fixed and earth-fixed velocity vectors is given by the transformation:

$$
\dot{\eta}=\mathbf{R}(\eta) \nu
$$

where $\mathbf{R}(\boldsymbol{\eta})$ is the rotation matrix defined by the formula:

$$
\mathbf{R}(\eta)=\mathbf{R}(\psi)=\left[\begin{array}{ccc}
\cos \psi & -\sin \psi & 0 \\
\sin \psi & \cos \psi & 0 \\
0 & 0 & 1
\end{array}\right]
$$

Notice that the matrix $\mathbf{R}(\psi)$ is non-singular for all $\psi$, hence $\mathbf{R}^{-1}(\psi)=\mathbf{R}^{\mathrm{T}}(\psi)$.

\section{b) Low frequency ship model}

For small velocities the low-frequency movements of the ship can be described using the following model [2]:

$$
\mathbf{M} \dot{v}+\mathbf{D} v=\tau+\mathbf{R}^{\mathrm{T}}(\eta) \mathbf{b}
$$

where $\tau \in \mathfrak{R}^{3}$ is the vector containing the control forces and the moment generated by the propulsion system consisting of the power thrusters and water jet propellers, while the non- 
modelled external forces and moment caused by the action of the wind, waves and sea currents are placed together into the bias component $\mathbf{b} \in \mathfrak{R}^{3}$ connected with the earth-fixed frame.

The matrix of inertia $\mathbf{M} \in \mathfrak{R}^{3 \times 3}$, which includes additional hydrodynamic inertia, is written as [2]:

$$
\mathbf{M}=\left[\begin{array}{ccc}
\mathrm{m}-\mathrm{X}_{\dot{\mathrm{u}}} & 0 & 0 \\
0 & \mathrm{~m}-\mathrm{Y}_{\dot{\mathrm{v}}} & \mathrm{mx}_{\mathrm{G}}-\mathrm{Y}_{\dot{\mathrm{r}}} \\
0 & \mathrm{mx}_{\mathrm{G}}-\mathrm{Y}_{\dot{\mathrm{r}}} & \mathrm{I}_{\mathrm{z}}-\mathrm{N}_{\dot{\mathrm{r}}}
\end{array}\right]
$$

where $m$ is the mass of the ship, $I_{z}$ is the moment of inertia around the $\mathrm{z}$ axis of the coordinate system connected with the vessel-fixed frame, and $\mathrm{x}_{\mathrm{G}}$ is the distance between the centre of gravity and the origin of the coord inate system connected with the moving ship, $\mathrm{X}_{\dot{\mathrm{u}}}, \mathrm{Y}_{\dot{\mathrm{v}}}, \mathrm{Y}_{\mathrm{i}}, \mathrm{N}_{\dot{\mathrm{v}}}$ and $\mathrm{N}_{\mathrm{r}}$ are the added masses connected with the accelerations along relevant axes.

The linear damping matrix $\mathbf{D} \in \mathfrak{R}^{3 \times 3}$ is defined as [2]:

$$
\mathbf{D}=\left[\begin{array}{ccc}
-\mathrm{X}_{\mathrm{u}} & 0 & 0 \\
0 & -\mathrm{Y}_{\mathrm{v}} & \mathrm{mu}_{0}-\mathrm{Y}_{\mathrm{r}} \\
0 & -\mathrm{N}_{\mathrm{v}} & \mathrm{mx}_{\mathrm{G}} \mathrm{u}_{0}-\mathrm{N}_{\mathrm{r}}
\end{array}\right]
$$

where the velocity of motion $\mathrm{u}_{0}=0$ in the dynamic positioning systems and $\mathrm{u}_{0}>0$ when the ship is in motion. Generally, the damping forces are nonlinear, but for the DP system the assumption about linear damping is satisfactory when the ship moves at low speed.

\section{c) First-order wave-induced model}

Modelling the ship movements generated by the waves of the first order is done based on the model of waves of the second order proposed by Balchen et al., [3] who used three harmonic oscillators. Saelid et al. improved the approximation of the WF wave model by introducing an additional coefficient [7]. This model (one for each degree of freedom) can be written as:

$$
h_{w}^{i}(s)=\frac{K_{w i} s}{s^{2}+2 \zeta_{i} \omega_{i} s+\omega_{i}^{2}}
$$

where $\omega_{\mathrm{i}}$ is the dominating frequency of the wave, $\xi_{\mathrm{i}}$ is the relative damping coefficient, and $\mathrm{K}_{\mathrm{wi}}$ is the parameter referring to the intensity of the wave. The above equation can be written in the state domain for three degrees of freedom as:

$$
\begin{gathered}
{\left[\begin{array}{l}
\dot{\xi}_{1} \\
\dot{\xi}_{2}
\end{array}\right]=\left[\begin{array}{cc}
\mathbf{0}_{3 \times 3} & \mathbf{I}_{3 \times 3} \\
-\boldsymbol{\Omega}^{2} & -2 \boldsymbol{\Lambda}
\end{array}\right] \cdot\left[\begin{array}{l}
\xi_{1} \\
\xi_{2}
\end{array}\right]+\left[\begin{array}{l}
\mathbf{0}_{3 \times 3} \\
\mathbf{K}_{\mathrm{w}}
\end{array}\right] \mathbf{w}_{\mathrm{w}}} \\
\eta_{\mathrm{w}}=\left[\begin{array}{ll}
\mathbf{0}_{3 \times 3} & \mathbf{I}_{3 \times 3}
\end{array}\right] \cdot\left[\begin{array}{c}
\xi_{1} \\
\xi_{2}
\end{array}\right]
\end{gathered}
$$

Here: $\mathbf{w}_{\mathrm{w}} \in \mathfrak{R}^{3}$ is the Gaussian white noise with zero mean value and $\boldsymbol{\eta}_{\mathrm{w}}=\left[\mathrm{x}_{\mathrm{w}}, \mathrm{y}_{\mathrm{w}}, \psi_{\mathrm{w}}\right]^{\mathrm{T}}$ describes the ship movements generated by the wave of the first order. $\Lambda=\operatorname{diag}\left\{\xi_{1} \omega_{1}, \xi_{2} \omega_{2}\right.$, $\left.\xi_{3} \omega_{3}\right\}, \Omega=\operatorname{diag}\left\{\omega_{1}, \omega_{2}, \omega_{3}\right\}$, and $\mathbf{K}=\operatorname{diag}\left\{\mathrm{K}_{\mathrm{w} 1}, \mathrm{~K}_{\mathrm{w} 2}, \mathrm{~K}_{\mathrm{w} 3}\right\}$ are the matrices of fixed dimensions. In compact notation:

$$
\begin{gathered}
\dot{\xi}=\mathbf{A}_{\mathrm{w}} \xi+\mathbf{E}_{\mathrm{w}} \mathbf{w}_{\mathrm{w}} \\
\eta_{\mathrm{w}}=\mathbf{C}_{\mathrm{w}} \xi
\end{gathered}
$$

\section{d) Bias modelling}

It is assumed that the forces and the moment connected with the non-modelled dynamics and external slowly-varying generated by the wind, waves and sea currents are used in bias model. The here adopted model is the Markov process of the first order.

$$
\dot{\mathbf{b}}=-\mathbf{T}^{-1} \mathbf{b}+\mathbf{E}_{\mathrm{b}} \mathbf{w}_{\mathbf{b}}
$$

where $\mathbf{b} \in \mathfrak{R}^{3}$ is the vector representing the slowly changing forces and moment, $\mathbf{w}_{\mathrm{b}} \in \mathfrak{R}^{3}$ is the vector of the Gaussian white noise with zero mean value, $\mathbf{T} \in \mathfrak{R}^{3 \times 3}$ is the diagonal matrix of positive time constants, and $\mathbf{E}_{\mathrm{b}} \in \mathfrak{R}^{3 \times 3}$ is the diagonal matrix scaling the noise amplitude $\mathbf{w}_{\mathrm{b}}$.

\section{e) Total system}

For conventional ships, the only available data are those referring to the measured ship position and heading data, therefore the equation relating the measured values has the form:

$$
\mathbf{y}=\eta+\eta_{\mathrm{w}}+\mathbf{v}
$$

where $\mathbf{v} \in \mathfrak{R}^{3}$ is the vector of the Gaussian measurement noise with zero mean value. To complete these data, the ship observer requires measurements of $\mathbf{u}$ to be able to calculate the longitudinal and lateral forces and the torque composing the vector $\tau$.

After collecting together all above equations we get the following model of the process:

$$
\begin{gathered}
\dot{v}=-\mathbf{M}^{-1} \mathbf{D} v+\mathbf{M}^{-1} \mathbf{R}^{\mathrm{T}}(\eta) \mathbf{b}+\mathbf{M}^{-1} \tau \\
\dot{\eta}=\mathbf{R}(\eta) v \\
\dot{\mathbf{b}}=-\mathbf{T}^{-1} \mathbf{b}+\mathbf{E}_{\mathrm{b}} \mathbf{w}_{\mathbf{b}} \\
\dot{\xi}=\mathbf{A}_{\mathrm{w}} \xi+\mathbf{E}_{\mathrm{w}} \mathbf{w}_{\mathrm{w}} \\
\mathbf{y}=\eta+\mathbf{C}_{\mathrm{w}} \xi+\mathbf{v}
\end{gathered}
$$

\section{THE EXTENDED KALMAN FILTER (EKF) DESIGN}

The design of the extended Kalman filter for a dynamic positioning system of ship motion control will be based on the nonlinear model having the form:

$$
\begin{gathered}
\dot{\mathbf{x}}=\mathbf{f}(\mathbf{x})+\mathbf{B u}+\mathbf{E w} \\
\mathbf{y}=\mathbf{H x}+\mathbf{v}
\end{gathered}
$$

where matrices $\mathbf{f}(\mathrm{x}), \mathbf{B}, \mathbf{E}$ and $\mathbf{H}$ are obtained from equations (11a)-(11e) and take the following forms:

$$
\begin{aligned}
& \mathbf{f}(\mathbf{x})=\left[\begin{array}{c}
\mathbf{R}(\eta) v \\
-\mathbf{M}^{-1} \mathbf{D} v+\mathbf{M}^{-1} \mathbf{R}^{\mathrm{T}}(\eta) \\
-\mathbf{T}^{-1} \\
\mathbf{A}_{\mathrm{w}} \xi
\end{array}\right] \quad \mathbf{B}=\left[\begin{array}{c}
\mathbf{0}_{3 \times 3} \\
\mathbf{M}^{-1} \\
\mathbf{0}_{3 \times 3} \\
\mathbf{0}_{6 \times 3}
\end{array}\right] \\
& \mathbf{E}=\left[\begin{array}{cc}
\mathbf{0}_{3 \times 3} & \mathbf{0}_{3 \times 3} \\
\mathbf{0}_{3 \times 3} & \mathbf{0}_{3 \times 3} \\
\mathbf{E}_{\mathrm{b}} & \boldsymbol{0}_{3 \times 3} \\
\mathbf{0}_{6 \times 3} & \mathbf{E}_{\mathrm{w}}
\end{array}\right] \\
& \mathbf{H}=\left[\begin{array}{llll}
\mathbf{I}_{3 \times 3} & \mathbf{0}_{3 \times 3} & \mathbf{0}_{3 \times 3} & \mathbf{C}_{\mathrm{w}}
\end{array}\right]
\end{aligned}
$$

Here: $\mathbf{x}=[\mathbf{v}, \boldsymbol{\eta}, \mathbf{b}, \xi]^{\mathrm{T}}, \mathbf{w}=\left[\mathbf{w}_{\mathbf{b}} \mathbf{w}_{\mathbf{w}}\right]^{\mathrm{T}}$ and $\mathbf{u}=\boldsymbol{\tau}$. The basic concept of the extended Kalman filter was proposed by Stanley Schmidt and sometimes this filter is referred to as the KalmanSchmidt filter [18] In the obtained model the nonlinearities are only included in the state equation which can be written in the following general form:

$$
\dot{\mathbf{x}}(\mathrm{t})=\mathbf{f}[\mathbf{x}(\mathrm{t}), \mathbf{u}(\mathrm{t})]+\mathbf{E} \mathbf{w}(\mathrm{t})
$$


In order to obtain the practically realisable algorithm of state vector estimation, the nonlinear function $\mathrm{f}[\mathbf{x}(\mathrm{t}), \mathbf{u}(\mathrm{t})]$ is expanded in the Taylor series about the current state vector estimate $\mathbf{x}=\hat{\mathbf{x}}$ :

$$
\begin{gathered}
\mathbf{f}[\mathbf{x}(\mathrm{t}), \mathbf{u}(\mathrm{t})]=\mathbf{f}[\hat{\mathbf{x}}(\mathrm{t}), \mathbf{u}(\mathrm{t})]+ \\
+\left.\frac{\partial \mathbf{f}[\mathbf{x}(\mathrm{t}), \mathbf{u}(\mathrm{t})]}{\partial \boldsymbol{x}(\mathrm{t})}\right|_{\mathrm{x}=\hat{\mathrm{x}}}[\mathbf{x}(\mathrm{t})-\hat{\mathbf{x}}(\mathrm{t})]+\ldots
\end{gathered}
$$

The above expansion can be done assuming that the nonlinear function has partial differentials. The linear approximation about the current equilibrium point is obtained by comparing all higher-order differences to zero:

$$
\begin{aligned}
& \mathbf{f}[\mathbf{x}(\mathrm{t}), \mathbf{u}(\mathrm{t})]-\mathbf{f}[\hat{\mathbf{x}}(\mathrm{t}), \mathbf{u}(\mathrm{t})]= \\
& =\left.\frac{\partial \mathbf{f}[\mathbf{x}(\mathrm{t}), \mathbf{u}(\mathrm{t})]}{\partial \boldsymbol{x}(\mathrm{t})}\right|_{\mathrm{x}=\hat{\mathrm{x}}}[\mathbf{x}(\mathrm{t})-\hat{\mathbf{x}}(\mathrm{t})]
\end{aligned}
$$

which leads to a linearized relation being the approximation of the equation (14):

$$
\frac{\mathrm{d}}{\mathrm{dt}} \Delta \mathbf{x}=\mathbf{A}(\mathrm{t}) \Delta \mathbf{x}+\mathbf{E w}(\mathrm{t})
$$

where:

$$
\mathbf{A}(\mathrm{t})=\left.\frac{\partial \mathbf{f}[\mathbf{x}(\mathrm{t}), \mathbf{u}(\mathrm{t})]}{\partial \mathbf{x}(\mathrm{t})}\right|_{\mathrm{x}=\hat{\mathrm{x}}}
$$

The extended Kalman filter is derived in the discrete form. For this purpose the linearized state equation (17) and the output equation (12b) are written in the following discrete form:

$$
\begin{gathered}
\mathbf{x}_{\mathrm{k}+1}=\mathbf{F}_{\mathrm{k}} \mathbf{x}_{\mathbf{k}}+\mathbf{G}_{\mathrm{k}} \mathbf{w}_{\mathbf{k}} \\
\mathbf{y}_{\mathbf{k}}=\mathbf{H} \mathbf{x}_{\mathbf{k}}+\mathbf{v}_{\mathrm{k}}
\end{gathered}
$$

where matrices $\mathbf{F}_{\mathrm{k}}$ and $\mathbf{G}_{\mathrm{k}}$ can be determined using the Euler forward method and the following relations:

$$
\begin{gathered}
\mathbf{F}_{\mathrm{k}}=\mathbf{I}_{\mathrm{n} \times \mathrm{n}}+\left.\mathrm{T} \frac{\partial \mathbf{f}_{\mathrm{k}}\left(\mathbf{x}_{\mathrm{k}}, \mathbf{u}_{\mathrm{k}}\right)}{\partial \mathbf{x}_{\mathrm{k}}}\right|_{\mathrm{x}_{\mathrm{k}}=\hat{\mathrm{x} k}} \underset{\mathbf{G}_{\mathrm{k}}=\mathrm{T} \cdot \mathbf{E}}{ }
\end{gathered}
$$

where $\mathrm{T}>0$ is the sampling period. This system includes $\mathrm{n}=15$ states, the process noise covariance weight matrix $\mathbf{Q}=\mathrm{E}\left(\mathbf{w}_{\mathrm{k}}^{\mathrm{T}} \mathbf{w}_{\mathbf{k}}\right) \in \mathfrak{R}^{\mathrm{n} \times \mathrm{n}}$ and the ship position and heading measurement noise covariance matrix $\mathbf{R}=\mathrm{E}\left(\mathbf{v}_{\mathrm{k}}^{\mathrm{T}} \mathbf{v}_{\mathbf{k}}\right) \in \mathfrak{R}^{3 \times 3}$. These matrices usually exist in the diagonal form. a detailed description of deriving the Kalman filter for the system described by equations (19a) and (19b) was given by Tomera [19]. Since in this case we deal with the exact nonlinear state equation (14), its discrete version will be used for calculating the process state values at time intervals between the sampling times. The measurements are done at the sampling times.

Finally we get the following algorithm of the extended Kalman filter.

The initial values:

$$
\begin{gathered}
\overline{\mathbf{x}}_{\mathrm{k}=0}=\mathbf{x}_{0} \\
\overline{\mathbf{P}}_{\mathrm{k}=0}=\mathrm{E}\left\{[\mathbf{x}(0)-\hat{\mathbf{x}}(0)] \cdot[\mathbf{x}(0)-\hat{\mathbf{x}}(0)]^{\mathrm{T}}\right\}=\mathbf{P}_{0}
\end{gathered}
$$

Corrector:

$$
\begin{gathered}
\mathbf{L}_{\mathrm{k}}=\overline{\mathbf{P}}_{\mathrm{k}} \mathbf{H}^{\mathrm{T}}\left[\mathbf{H} \overline{\mathbf{P}}_{\mathrm{k}} \mathbf{H}^{\mathrm{T}}+\mathbf{R}\right]^{-1} \\
\hat{\mathbf{P}}_{\mathrm{k}}=\left(\mathbf{I}-\mathbf{L}_{\mathrm{k}} \mathbf{H}\right) \overline{\mathbf{P}}_{\mathrm{k}}\left(\mathbf{I}-\mathbf{L}_{\mathrm{k}} \mathbf{H}\right)^{\mathrm{T}}+\mathbf{L}_{\mathrm{k}} \mathbf{R} \mathbf{L}_{\mathrm{k}}^{\mathrm{T}} \\
\hat{\mathbf{x}}_{\mathrm{k}}=\overline{\mathbf{x}}_{\mathrm{k}}+\mathbf{L}_{\mathrm{k}}\left(\mathbf{y}_{\mathrm{k}}-\mathbf{H} \overline{\mathbf{x}}_{\mathrm{k}}\right)
\end{gathered}
$$

Predictor:

$$
\begin{gathered}
\overline{\mathbf{P}}_{\mathrm{k}+1}=\mathbf{F}_{\mathrm{k}} \hat{\mathbf{P}}_{\mathrm{k}} \mathbf{F}_{\mathrm{k}}^{\mathrm{T}}+\mathbf{G}_{\mathrm{k}} \mathbf{Q} \mathbf{G}_{\mathrm{k}}^{\mathrm{T}} \\
\overline{\mathbf{x}}_{\mathrm{k}+1}=\mathbf{f}_{\mathrm{k}}\left(\hat{\mathbf{x}}_{\mathrm{k}}, \mathbf{u}_{\mathrm{k}}\right)
\end{gathered}
$$

where $\hat{\mathbf{x}}_{\mathrm{k}}=\left[v_{\mathrm{k}}^{\mathrm{T}}, \hat{\eta}_{\mathrm{k}}^{\mathrm{T}}, \hat{\mathbf{b}}_{\mathrm{k}}^{\mathrm{T}}, \hat{\xi}_{\mathrm{k}}^{\mathrm{T}}\right]^{\mathrm{T}}$ is the estimated state vector, matrices $\mathbf{F}_{k}$ and $\mathbf{G}_{k}$ are determined from formulas (20a) and (20b), and the discrete nonlinear function $\mathbf{F}_{\mathrm{k}}\left(\hat{\mathbf{x}}_{\mathrm{k}}, \mathbf{u}_{\mathrm{k}}\right)$ can be determined using the Euler forward method:

$$
\overline{\mathbf{x}}_{\mathrm{k}+1}=\mathbf{f}_{\mathrm{k}}\left(\hat{\mathbf{x}}_{\mathrm{k}}, \mathbf{u}_{\mathrm{k}}\right)=\hat{\mathbf{x}}_{\mathrm{k}}+\mathrm{T}\left[\mathbf{f}\left(\hat{\mathbf{x}}_{\mathrm{k}}\right)+\mathbf{B} \mathbf{u}_{\mathrm{k}}\right]
$$

where $\mathrm{T}>0$ is the sampling period.

\section{NONLINEAR OBSERVER DESIGN}

The here presented nonlinear observer is similar to that proposed by Fossen and Strand and used in the dynamic positioning system (Fossen and Strand, 1999). The stability of the nonlinear observers is checked using the Lapunov analysis. For this purpose certain assumptions concerning the mathematical model of the process dynamics are to be made:

a) The bias model (11c) and the wave model (11d) include the Gaussian white noises with zero mean component. In the Lapunov analysis these terms are to be neglected.

b) Also neglected are the noises of ship position and heading measurements, i.e. $\mathbf{v}=\mathbf{0}$, as these terms are of little influence compared to the ship movements caused by the waves.

c) It is assumed that the amplitude of the movements generated by the waves $\psi_{\mathrm{w}}$ is of negligible importance and is equal to less than 1 degree during regular ship operation and less to 5 degrees in extreme weather conditions. Hence $\mathbf{R}(\boldsymbol{\eta})=$ $=\mathbf{R}(\psi) \approx \mathbf{R}\left(\psi+\psi_{\mathrm{w}}\right)$. It results from condition (b) that $\mathbf{R}(\psi) \approx$ $\approx \mathbf{R}\left(\psi_{\mathrm{y}}\right)$, where $\psi_{\mathrm{y}}=\psi+\psi_{\mathrm{w}}$ represents the measured ship heading.

d) The following model properties are assumed for the inertia and damping matrices

$$
\mathbf{M}=\mathbf{M}^{\mathrm{T}}>0, \dot{\mathbf{M}}=0, \mathbf{D}>0
$$

After taking into account assumptions (a-d), the equations (11a)-(11e) describe the following mathematical model of the process [11]:

$$
\begin{gathered}
\mathbf{M} \dot{v}=-\mathbf{D} v+\mathbf{R}^{\mathrm{T}}\left(\psi_{\mathbf{y}}\right) \mathbf{b}+\tau \\
\dot{\eta}=\mathbf{R}\left(\psi_{\mathrm{y}}\right) v \\
\dot{\mathbf{b}}=-\mathbf{T}^{-1} \mathbf{b} \\
\dot{\xi}=\mathbf{A}_{\mathrm{w}} \xi \\
\mathbf{y}=\eta+\mathbf{C}_{\mathrm{w}} \xi
\end{gathered}
$$

This model is used for designing the nonlinear observer.

\section{a) Equations of the observer}

The nonlinear observer based on the model of the ship and disturbances (25a)-(25e) is described by the following equations:

$$
\begin{gathered}
\mathbf{M} \dot{\hat{v}}=-\mathbf{D} \hat{v}-\mathbf{R}^{\mathrm{T}}\left(\psi_{\mathrm{y}}\right) \hat{\mathbf{b}}+\boldsymbol{\tau}+\mathbf{R}^{\mathrm{T}}\left(\psi_{\mathrm{y}}\right) \mathbf{K}_{1} \widetilde{\mathbf{y}} \\
\dot{\hat{\eta}}=\mathbf{R}\left(\psi_{\mathrm{y}}\right) \cdot \hat{v}+\mathbf{K}_{2} \widetilde{\mathbf{y}} \\
\dot{\hat{\mathbf{b}}}=-\mathbf{T}_{\mathrm{b}}^{-1} \hat{\mathbf{b}}+\mathbf{K}_{3} \widetilde{\mathbf{y}} \\
\dot{\hat{\xi}}=\mathbf{A}_{\mathrm{w}} \xi+\mathbf{K}_{\mathbf{4}} \widetilde{\mathbf{y}}
\end{gathered}
$$




$$
\hat{\mathbf{y}}=\eta+\mathbf{C}_{w} \xi
$$

where $\widetilde{\mathbf{y}}=\mathbf{y}-\hat{\mathbf{y}} \in \mathfrak{R}^{3}$ is the estimation error, $\mathbf{K}_{1}, \mathbf{K}_{2}, \mathbf{K}_{3} \in \mathfrak{R}^{3 \times 3}$ and $\mathbf{K}_{4} \in \mathfrak{R}^{3 \times 3}$ are the observer amplification matrices.

\section{b) Observer estimation errors}

The observer estimation errors are defined as $\widetilde{v}=v-\hat{v}$, $\widetilde{\eta}=\eta-\hat{\eta}, \widetilde{\mathbf{b}}=\mathbf{b}-\hat{\mathbf{b}}$ and $\widetilde{\xi}=\xi-\hat{\xi}$. Hence:

$$
\begin{gathered}
\mathbf{M} \dot{\widetilde{v}}=-\mathbf{D} \tilde{v}+\mathbf{R}^{\mathrm{T}}\left(\psi_{\mathrm{y}}\right) \widetilde{\mathbf{b}}-\mathbf{R}^{\mathrm{T}}\left(\psi_{\mathrm{y}}\right) \mathbf{K}_{1} \widetilde{\mathbf{y}} \\
\dot{\tilde{\eta}}=\mathbf{R}\left(\psi_{\mathrm{y}}\right) \cdot \widetilde{v}-\mathbf{K}_{2} \widetilde{\mathbf{y}} \\
\dot{\widetilde{\mathbf{b}}}=-\mathbf{T}_{\mathrm{b}}^{-1} \widetilde{\mathbf{b}}-\mathbf{K}_{3} \widetilde{\mathbf{y}}+\mathbf{E}_{\mathrm{b}} \mathbf{w}_{\mathrm{b}} \\
\dot{\vec{\xi}}=\mathbf{A}_{\mathrm{w}} \widetilde{\xi}-\mathbf{K}_{4} \widetilde{\mathbf{y}}+\mathbf{E}_{\mathrm{w}} \mathbf{w}_{\mathrm{w}} \\
\tilde{\mathbf{y}}=\widetilde{\eta}+\mathbf{C}_{\mathrm{w}} \xi
\end{gathered}
$$

After defining the new output:

$$
\widetilde{\mathbf{z}}_{\mathrm{o}}=\mathbf{K}_{1} \tilde{\mathbf{y}}-\mathbf{b}=\mathbf{C}_{\mathrm{o}} \widetilde{\mathbf{x}}_{\mathrm{o}}
$$

and vectors:

$$
\widetilde{\mathbf{x}}_{\mathrm{o}}=\left[\begin{array}{c}
\widetilde{\eta} \\
\widetilde{\mathbf{b}} \\
\widetilde{\xi}
\end{array}\right] \quad \mathbf{w}=\left[\begin{array}{c}
\mathbf{w}_{\mathrm{b}} \\
\mathbf{w}_{\mathrm{w}}
\end{array}\right]
$$

the error dynamics (27a)-(27e) can be written in a compact form as:

$$
\begin{gathered}
\mathbf{M} \dot{\widetilde{\mathbf{v}}}=-\mathbf{D} \widetilde{\mathbf{v}}+\mathbf{R}^{\mathrm{T}}\left(\psi_{\mathrm{y}}\right) \mathbf{C}_{\mathrm{o}} \mathbf{x}_{\mathrm{o}}=-\mathbf{D} \widetilde{\mathbf{v}}+\mathbf{R}^{\mathrm{T}}\left(\psi_{\mathrm{y}}\right) \mathbf{z}_{\mathrm{o}} \\
\dot{\widetilde{\mathbf{x}}}_{\mathrm{o}}=\mathbf{A}_{\mathrm{o}} \mathbf{x}_{\mathrm{o}}+\mathbf{B}_{\mathrm{o}} \mathbf{R}\left(\psi_{\mathrm{y}}\right) \widetilde{\mathbf{v}}+\mathbf{E}_{\mathrm{o}} \mathbf{w}
\end{gathered}
$$

where:

$$
\begin{array}{r}
\mathbf{A}_{\mathrm{o}}=\left[\begin{array}{ccc}
-\mathbf{K}_{2} & \mathbf{0}_{3 \times 3} & -\mathbf{K}_{2} \mathbf{C}_{\mathrm{w}} \\
-\mathbf{K}_{3} & -\mathbf{T}_{\mathrm{b}}^{-1} & -\mathbf{K}_{3} \mathbf{C}_{\mathrm{w}} \\
-\mathbf{K}_{4} & \mathbf{0}_{6 \times 3} & \mathbf{A}_{\mathrm{w}}-\mathbf{K}_{4} \mathbf{C}_{\mathrm{w}}
\end{array}\right] \\
\mathbf{C}_{\mathrm{o}}=\left[\begin{array}{ll}
\mathbf{K}_{1}-\mathbf{I}_{3 \times 3} & \mathbf{K}_{1} \mathbf{C}_{\mathrm{w}}
\end{array}\right] \\
\mathbf{B}_{\mathrm{o}}=\left[\begin{array}{c}
\mathbf{I}_{3 \times 3} \\
\mathbf{0}_{3 \times 3} \\
\mathbf{0}_{6 \times 3}
\end{array}\right] \quad \mathbf{E}_{\mathrm{o}}=\left[\begin{array}{cc}
\mathbf{0}_{3 \times 3} & \mathbf{0}_{3 \times 3} \\
\mathbf{E}_{\mathrm{b}} & \mathbf{0}_{3 \times 3} \\
\mathbf{0}_{6 \times 3} & \mathbf{E}_{\mathrm{w}}
\end{array}\right]
\end{array}
$$

The observer amplification matrices can be selected in such a way that the error dynamics are passive.

\section{SIMULATION AND EXPERIMENTAL RESULTS}

To assess the quality and robustness of the designed observers, the simulation tests and experimental investigations were done. The experimental investigations were performed on the lake on which the waves are so short that they are of no importance for the ship motion control process, which made it impossible to test the quality of observers' operation at the presence of waves. That is why the equations modelling the behaviour of waves were not included to the models of the observers. As a consequence, the number of the estimated state variables was equal to $\mathrm{n}=9$.

The investigations were performed using the training ship "Blue Lady" owned by the Foundation of Sailing Safety and Environment Protection in Ilawa and used for training navigators. The ship "Blue Lady" is an autonomous model of the VLCC (Very Large Crude Carrier) class tanker designed for transporting crude oil. The model, made of epoxide laminate in scale 1:24, is equipped with a set of propellers with electric motors fed from accumulator batteries. The overall length of Blue Lady is $\mathrm{L}=13.75 \mathrm{~m}$, the width is $\mathrm{B}=2.38 \mathrm{~m}$, and the mass is $\mathrm{m}=22.934 \times 10^{3} \mathrm{~kg}$ [20]. The moving coordinate system is fixed to the centre of gravity.

The designed observers base on the following mathematical model of "Blue Lady".

$$
\begin{gathered}
\mathbf{M}=\operatorname{diag}(23664.924830 .6455182 .1) \\
\mathbf{D}=\left[\begin{array}{ccc}
21.049 & 0 & 0 \\
0 & 259.8 & -855.4 \\
0 & -855.4 & 6130.5
\end{array}\right]
\end{gathered}
$$

The time constants for the bias were selected in the following way:

$$
\mathbf{T}=\operatorname{diag}(100010001000)
$$

For the extended Kalman filter the following matrices were selected:

- the process noise covariance matrix:

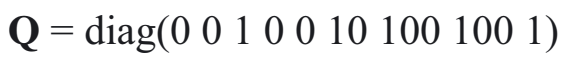

- the ship position and heading measurement noise covariance matrix:

$$
\mathbf{R}=\operatorname{diag}\left(\begin{array}{lll}
0.01 & 0.010 .01
\end{array}\right)
$$

The amplification matrices for the nonlinear observer were determined as:

$$
\begin{gathered}
\mathbf{K}_{1}=\operatorname{diag}\left(\begin{array}{lll}
1000 & 1000 & 1000) \\
\mathbf{K}_{2}=\operatorname{diag}(0.510 .5 & 0.025)
\end{array}\right. \\
\mathbf{K}_{3}=\operatorname{diag}\left(\begin{array}{llll}
100 & 100 & 10
\end{array}\right)
\end{gathered}
$$

Both the simulation tests and experimental investigations were performed using the recording frequency equal to $1 \mathrm{~Hz}$.

Firstly, the simulation tests were performed in the calculating environment Matlab/Simulink based on the mathematical model of Blue Lady described in detail by Gierusz [21, 22]. These investigations were performed at the presence of measurement noises which were added to the posiotions and hesding measurements in simulations at the presence of the external disturbances. In simulation study assumed that on ship was acting wind with average speed equal $2 \mathrm{~m} / \mathrm{s}$ in directions 0 degrees. The simulation results are shown in Figs. $2-4$. The actual and estimated velocities in surge, sway and yaw are shown in the bottom of plots.

The observer algorithms which underwent simulation tests were then implemented on the training ship "Blue Lady" on the lake Silm near Ilawa. The performed experimental investigations aimed at assessing the quality of observers' operation and their resistance to disturbances. During the investigations the ship position coordinates $(\mathrm{x}, \mathrm{y})$ were measured by the GPS system, while the ship heading $\psi$ was recorded by the gyro-compass. The velocities: surge $\mathrm{u}$, sway $\mathrm{v}$ and yaw $\mathrm{r}$ were estimated by the observers. Figures $5-7$ show sample results of ship movement using three propulsion systems: the bow tunnel thruster $(n=0.5)$, the stern tunnel thruster $(n=0.5)$ and the propeller screw $(\mathrm{n}=100 \mathrm{rev} / \mathrm{min})$. The settings of the tunnel thrusters can be set within the range of $\langle-1 \ldots+1\rangle$.

The state variables which were estimated by the extended Kalman filter are shown in the left-hand columns in Figs. 6 - 7, 
while those estimated by the nonlinear observer are collected in the right-hand column. The curves representing the measured and estimated elements of the vector $\boldsymbol{\eta}=[\mathrm{x}, \mathrm{y}, \psi]^{\mathrm{T}}$ are smooth lines. The situation is different for the estimated velocity vector $\mathrm{v}=[\mathrm{u}, \mathrm{v}, \mathrm{r}]^{\mathrm{T}}$, whose diagrams reveal certain noise in case of the extended Kalman filter while the estimates calculated by the nonlinear observer are still smooth.
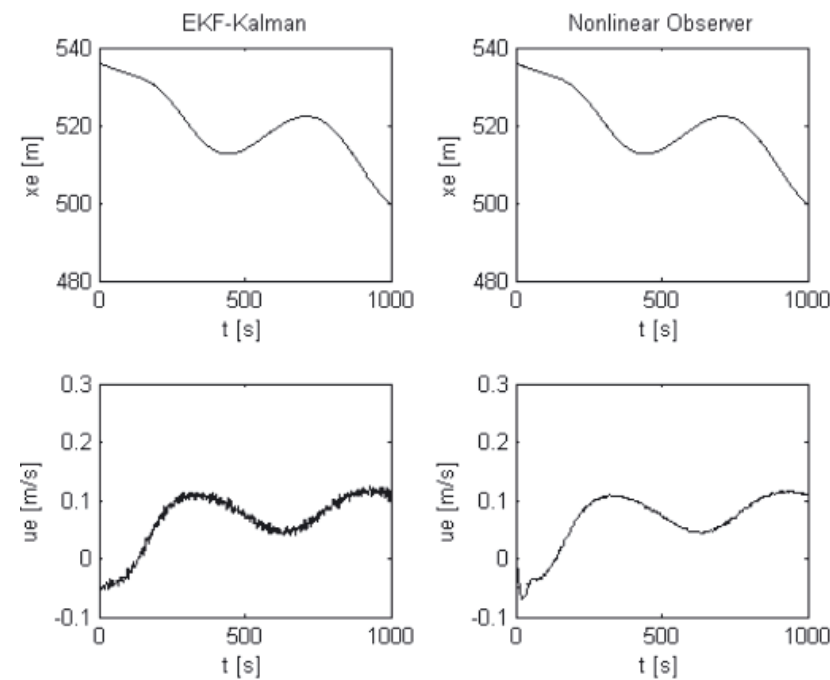

Fig. 2. Simulation study: actual position (dotted) with estimate (solid) and actual velocity $u$ in surge (dotted) and estimate (solid)
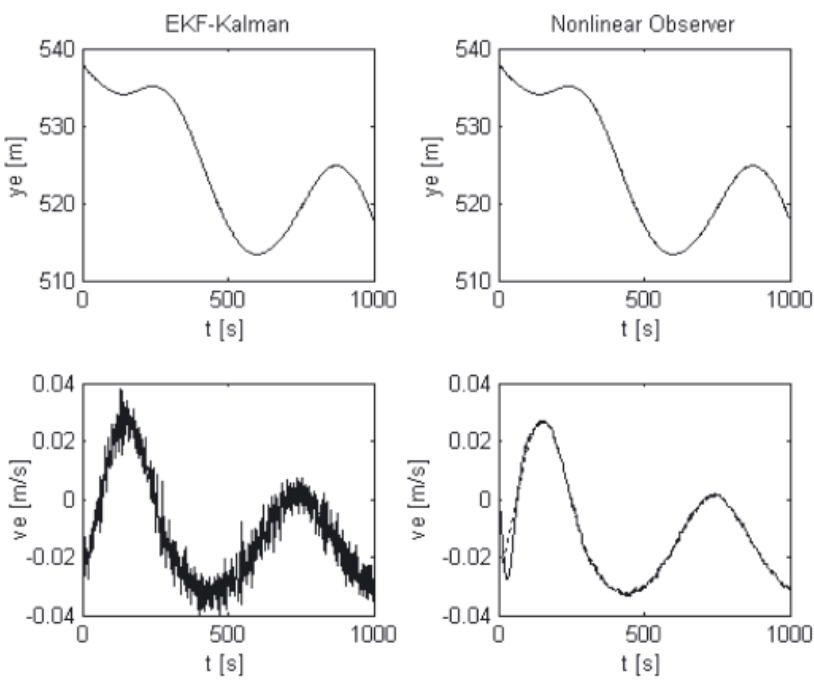

Fig. 3. Simulation study: actual position (dotted) with estimate (solid) and actual velocity $v$ in sway (dotted) and estimate (solid)
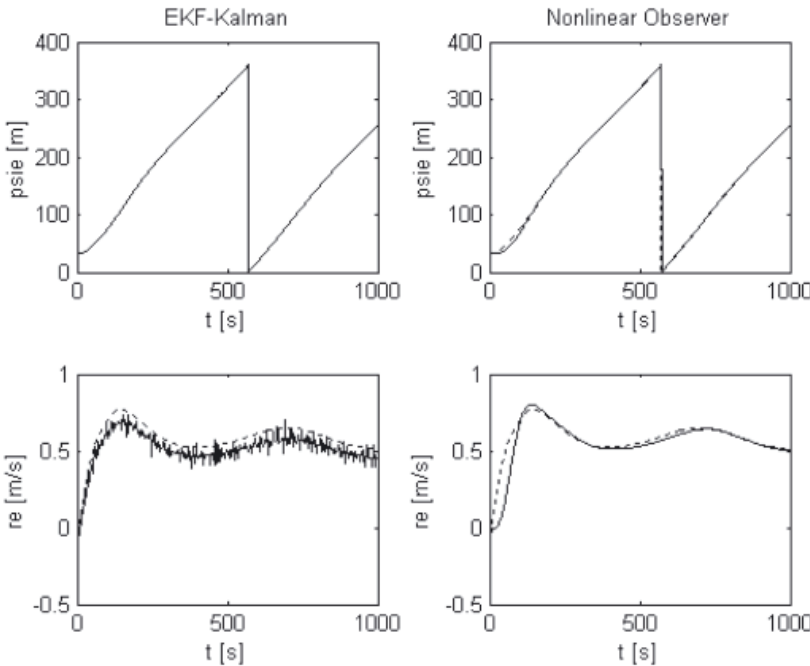

Fig. 4. Simulation study: actual heading angle $\psi$ (dotted) with estimate (solid) and actual angular rate $r$ in yaw (dotted) with estimate (solid)
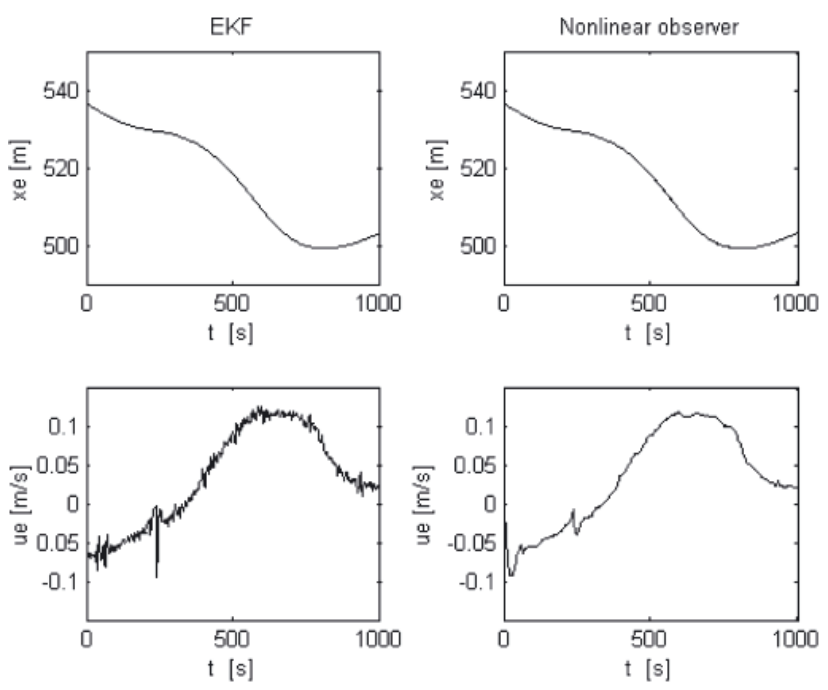

Fig. 5. Experimental data: measured position with estimate and estimated velocity $u$ in surge. Left-hand column-extended Kalman filter, right-hand column - nonlinear observer
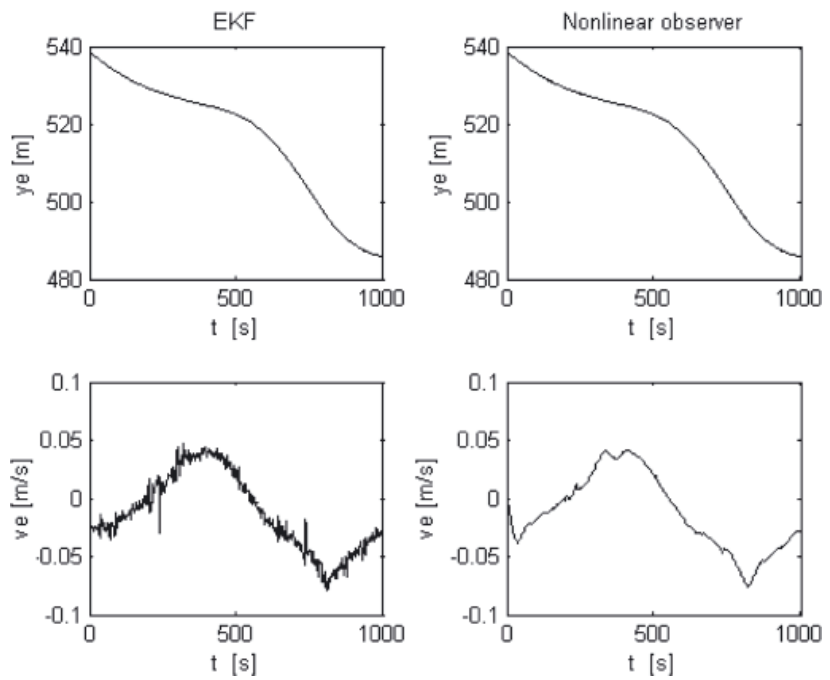

Fig. 6. Experimental data: measured position with estimate and estimated velocity $v$ in sway. Left-hand column - extended Kalman filter, right-hand column - nonlinear observer
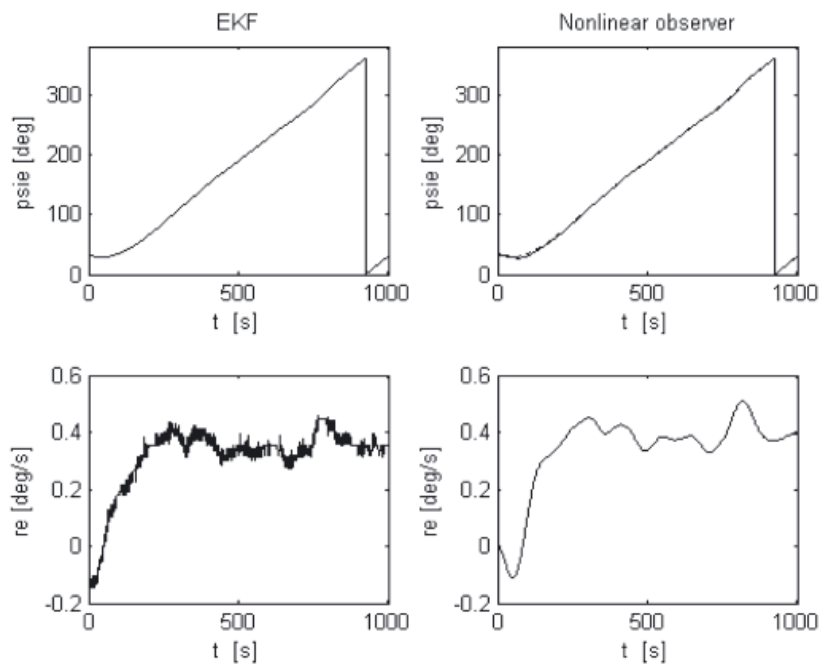

Fig. 7. Experimental data: measured heading angle $\psi$ with estimate and estimated angular rate $r$ in yaw. Left-hand column - extended Kalman filter, right-hand column - nonlinear observer. When estimating the ship motion velocity in all three degrees of freedom, we can observe the difference between the values estimated for the initial time, after switching the observers on. When starting the experimental test, the values returned by the nonlinear observer start from zero, while the extended Kalman filter detects, from the very beginning, the initial values of the ship motion velocity 


\section{REMARKS AND CONCLUSIONS}

- The article presents two algorithms of observers for estimating ship motion parameters in three degrees of freedom: surge, sway and yaw. The observers were first tested numerically via simulations done on a complex nonlinear computer model of the training ship "Blue Lady".

- The simulation tests made it possible to determine the parameters of the examined observers.

Based on the satisfactory results of the simulation tests, the next step of investigations was the use of the examined observers for estimating the parameters of the real model of "Blue Lady" sailing on the lake Silm in Ilawa-Kamionka. The obtained results of the experimental investigations are presented in the article. Comparing the obtained results we can easily notice that the time-histories of the velocities estimated by the nonlinear observer are smooth and do not reveal noise, while those generated by the extended Kalman filter in all directions of motion reveal the measuring noise.

- The designed observers can be used in dynamic multivariable systems controlling the motion of ships with a number of propeller systems, including such actions as dynamic positioning, tracking, or mooring. Further research work will be focused on designing a nonlinear dynamic positioning control system making use of the nonlinear observer.

\section{BIBLIOGRAPHY}

1. Fossen, T. I.: Marine Control Systems: Guidance, Navigation, and Control of Ships, Rigs and Underwater Vehicles, Marine Cybernetics, Trondheim, Norway, 2002.

2. Fossen, T. I.: Guidance and Control of Ocean Vehicles, John Wiley \& Sons Ltd, Chichester, England, 1994.

3. Balchen, J. G., Jenssen, N. A., Saelid. S.: Dynamic positioning using Kalman filtering and optimal control theory. In IFAC/IFIP Symposium on Automation in Offshore Oil Field Operation. Bergen, Norway, pp. 183-186, 1976.

4. Balchen, J. G., Jenssen, N. A., Saelid, S.: Dynamic positioning of floating vessels based on Kalman filtering and optimal control, Proceedings of the $19^{\text {th }}$ IEEE Conference on Decision and Control, New York, pp. 852-864, 1980.

5. Balchen, J. G., Jenssen, N. A., Mathiasen, E., Saelid, S.: a dynamic positioning system based on Kalman filtering and optimal control, Modeling, Identification and Control, Vol. 1, No 3, pp. 135-163, 1980

6. Fung, P., Grimble, M.: Dynamic Ship Positioning Using SelfTuning Kalman Filter. IEEE Transactions on Automatic Control, Vol. 28, No 3, pp. 339-349, 1983.

7. Saelid, S., Jensen, N. A., Balchen, J. G.: Design and analysis of a dynamic positioning system based on Kalman filtering and optimal control, IEEE Transactions on Automatic Control, Vol. 28, No 3, pp. 331-339, 1983.

8. Sorensen, A. J., Sagatun, S. I., Fossen, T. I.: Design of a Dynamic Position System Using Model-based Control., Control Engeenering Practice, Vol. 4, No 3, pp. :359-368, 1996.
9. Strand, J. P., Sorensen, A. J., Fossen, T. I.: Modelling and control of thruster assisted position mooring systems for ships, Proceedings of IFAC Manoeuvering and Control of Marine Craft (MCMC'97), Brijuni, Croatia, pp. 160-165, 1997.

10.Fossen, T. I., Grovlen, A.: Nonlinear output feedback control of dynamic positioned ships using vectorial observer backstepping, IEEE Transactions on Control Systems Technology, Vol. 6, No 1, pp. 121-128, 1998.

11.Fossen, T. I., Strand, J. P.: Passive nonlinear observer design for ships using Lyapunov methods: Experimental results with a supply vessel, Automatica, Vol. 35, No 1, pp. 3-16, 1999.

12.Loria, A., Fossen, T. I., Panteley, E.: a Separation Principle for Dynamic Positioning of Ships: Theoretical and Experimental Results, IEEE Transactions on Control Systems Technology, Vol. 8, No 2, pp. 332-343, 2000.

13.Krstic, M., Kanellakopoulos, I., Koktovic, P.: Nonlinear and Adaptive Control Design, John Wiley \& Sons, 1995.

14.Kim, M. H., Inman, D. J.: Development of a robust non-linear observer for dynamic positioning of ships, Proceedings of the Institution of Mechanical Engineers Part I: Journal of Systems and Control Engineering, Vol. 218, No 1, pp. 1-11, 2004.

15.Snijders, J. G., van der Woude, J. W., Westhuis, J.: Nonlinear Observer Design for Dynamic Positioning, Proceedings of Dynamic Positioning Conference, Houston US, November, pp. $15-16,2000$

16.Hajivand, A., Mousavizadegan, S. H.: The effect of memory in passive nonlinear observer design for a DP system, Proceedings of the Dynamic Positioning Conference, Houston, US, 2010.

17.Sorensen, A.: Marine Cybernetics: Modelling and Control, $5^{\text {th }}$ edn, Norwegian University of Science \& Technology, Department Engineering Cybernetics, Trondheim, Norway, 2005.

18.Bellantoni, J. F., Dodge, K. W.: a square root formulation of the Kalman-Schmidt filter, AIAA Journal, Vol. 5, No 7, pp. 13091314, 1967.

19.Tomera M.: Discrete Kalman filter design for multivariable ship motion control: experimental results with training ship, Joint Proceedings of Gdynia Maritime Academy \& Hochschule Bremerhaven, Bremerhaven, pp. 26-34, 2010.

20. Website of the Foundation for Safety of Navigation and Environment Research, 2010. Available: http://www.ilawashiphandling.com.pl

21. Gierusz, W.: Simulation model of the shiphandling training boat Blue Lady. Proceedings of Control Applications in Marine Systems. Glasgow, Scotland, UK, 2001.

22. Gierusz, W.: Synthesis of multivariable systems of precise ship motion control with the aid of selected resistant system design methods. Publishing House of Gdynia Maritime University, (in Polish), 2005.

\section{CONTACT WITH THE AUTHOR}

Mirosław Tomera, Ph. D.

Faculty of Marine Electrical Engineering, Gdynia Maritime University, Morska 81-87

81-225 Gdynia, POLAND

e-mail: tomera@am.gdynia.pl 